\title{
Results on Cumulative Measure of Inaccuracy in Record Values
}

\author{
Saeid Tahmasebi * \\ Department of Statistics, Persian Gulf University, Bushehr, Iran \\ tahmasebi@pgu.ac.ir \\ Ahmad Nezakati \\ Department of Statistics, Shahrood University of Technology, Iran \\ nezakati@shahroodut.ac.ir \\ Safeih Daneshi \\ Department of Statistics, Shahrood University of Technology, Iran \\ sdaneshi445@gmail.com
}

Received 24 March 2016

Accepted 19 November 2017

\begin{abstract}
In this paper, we propose cumulative measure of inaccuracy in lower record values and study characterization results in case of dynamic cumulative inaccuracy. We also discuss some properties of the proposed measures. Finally, we study a problem of estimating the cumulative measure of inaccuracy by means of the empirical cumulative inaccuracy in lower record values.
\end{abstract}

Keywords: Measure of inaccuracy; Cumulative inaccuracy; Record values.

2000 Mathematics Subject Classification: 62N05, 90B25

\section{Introduction}

Let $X$ and $Y$ be two non-negative random variables with distribution functions $F(x), G(x)$ and reliability functions $\bar{F}(x), \bar{G}(x)$, respectively. If $f(x)$ is the actual probability density function(pdf) corresponding to the observations and $g(x)$ is the density assigned by the experimenter, then the inaccuracy measure of $X$ and $Y$ is defined by Kerridge(1961) as

$$
I(X, Y)=I(f, g)=-\int_{0}^{+\infty} f(x) \log g(x) d x .
$$

It has applications in statistical inference, estimation and coding theory. Analogous to the Kerridge measure of inaccuracy (1.1), Thapliyal and Taneja (2015a) proposed a cumulative inaccuracy measure as

$$
I(F, G)=-\int_{0}^{+\infty} F(x) \log G(x) d x .
$$

${ }^{*}$ Corresponding 
Tahmasebi, Nezakati and Daneshi

The mean inactivity time (MIT) function is of interest in many fields such as reliability, survival analysis, actuarial studies, etc. MIT of a random variable $X$ is defined as

$$
\mu_{1}(t)=E(t-X \mid X<t) .
$$

Ghosh and Kundu(2017) obtained a connection between $\mu_{1}(t)$ and $I(F, G)$ as

$$
I(F, G)=E\left(\frac{\mu_{1}(Y) F(Y)}{G(Y)}\right) .
$$

Let $X_{1}, X_{2}, \ldots$ be a sequence of iid random variables having an absolutely cdf $\mathrm{F}(\mathrm{x})$ and $\mathrm{pdf} f(\mathrm{x})$. An observation $X_{j}$ is called a lower record value if its value is less than that of all previous observations. Thus, $X_{j}$ is a lower record if $X_{j}<X_{i}$ for every $i<j$. An analogous definition can be given for upper record values. Then the record times sequence $T_{n}, n \geq 1$ is defined in the following manner: $T_{1}=1$, with probability 1 , and for $n \geq 2, T_{n}=\min \left\{j: j>T_{n-1}, X_{j}<X_{T_{n-1}}\right\}$. The lower record value sequence can be defined by $L_{n}=X_{T_{n}}, n \geq 1$. Then the density function and cdf of $L_{n}$, which are denoted by $f_{L_{n}}$ and $F_{L_{n}}$, respectively, are given by

$$
\begin{gathered}
f_{L_{n}}(x)=\frac{[-\log F(x)]^{n-1}}{(n-1) !} f(x), \\
F_{L_{n}}(x)=\sum_{j=0}^{n-1} \frac{[-\log F(x)]^{j}}{j !} F(x) .
\end{gathered}
$$

Record values are applied in problems such as industrial stress testing, meteorological analysis, hydrology, sporting and economics. In reliability theory, records values are used to study, for example, technical systems which are subject to shocks, e.g. peaks of voltages. For more details about records and their applications, one may refer to Arnold et al.(1992). Several authors have worked on measures of inaccuracy for ordered random variables. Thapliyal and Taneja (2013) proposed the measure of inaccuracy between the ith order statistic and the parent random variable. Thapliyal and Taneja (2015a)developed measures of dynamic cumulative residual and past inaccuracy. They studied characterization results of these dynamic measures under proportional hazard model and proportional reversed hazard model. Recently Thapliyal and Taneja (2015b) have introduced the measure of residual inaccuracy of order statistics and prove a characterization result for it. In this paper we propose cumulative past measure of inaccuracy and study their characterization results. The paper is organized as follows: In Section 2, we consider a measure of inaccuracy associated with $F_{L_{n}}$ and $F$ and obtain some results of its properties. In Section 3, we study dynamic version of inaccuracy associated with $F_{L_{n}}$ and $F$. In Section 4, we propose empirical cumulative measure of inaccuracy in lower record values. Throughout the paper we assume that the terms increasing and decreasing are used in non-strict sense.

\section{Cumulative measure of inaccuracy}

The cumulative measure of inaccuracy between $F_{L_{n}}$ (distribution function of nth lower record value $L_{n}$ ) and $F$ is presented as

$$
I\left(F_{L_{n}}, F\right)=-\int_{0}^{\infty} F_{L_{n}}(x) \log F(x) d x
$$




$$
\begin{aligned}
& =-\int_{0}^{\infty} F(x) \sum_{j=0}^{n-1} \frac{[-\log F(x)]^{j}}{j !} \log F(x) d x \\
& =\sum_{j=0}^{n-1} \int_{0}^{\infty} \frac{[-\log F(x)]^{j+1}}{j !} F(x) d x \\
& =\sum_{j=0}^{n-1}(j+1) \int_{0}^{\infty} \frac{[-\log F(x)]^{j+1}}{(j+1) !} f(x) \frac{1}{\tilde{\lambda}(x)} d x \\
& =\sum_{j=0}^{n-1}(j+1) E_{L_{j+2}}\left[\frac{1}{\tilde{\lambda}(X)}\right],
\end{aligned}
$$

where $\tilde{\lambda}(x)=\frac{f(x)}{F(x)}$ is the reversed hazard rate and $L_{j+2}$ is a random variable with density function $f_{L_{j+2}}(x)=\frac{[-\log F(x)]^{j+1} f(x)}{(j+1) !}$.

In the following, we present some examples and properties of $I\left(F_{L_{n}}, F\right)$.

\section{Example 2.1.}

i. If $X$ has a inverse Weibull distribution with the $\operatorname{cdf} F(x)=\exp \left(-\left(\frac{\alpha}{x}\right)^{\beta}\right), x>0$. Then, we have

$$
I\left(F_{L_{n}}, F\right)=\frac{\alpha}{\beta} \sum_{j=0}^{n-1} \frac{\Gamma\left(\frac{(j+1) \beta-1}{\beta}\right)}{j !} .
$$

Figure 1 shows the function $I\left(F_{L_{n}}, F\right)$ for $\alpha=\beta=2$. It is an increasing function of $n$. ii. If $X$ is uniformly distributed in $[0, \theta]$. Then, we obtain

$$
I\left(F_{L_{n}}, F\right)=\theta \sum_{j=0}^{n-1}(j+1)\left(\frac{1}{2}\right)^{j+1} .
$$

From Figure 1 it is clear that $I\left(F_{L_{n}}, F\right)$ for standard uniform distribution is increasing function of $n$ and $\lim _{n \rightarrow \infty} I\left(F_{L_{n}}, F\right)=2 \theta$.

iii. If $X$ is exponentially distributed with mean $\frac{1}{\lambda}$. Then, we obtain

$$
I\left(F_{L_{n}}, F\right)=\frac{1}{\lambda} \sum_{j=0}^{n-1} \sum_{k=0}^{\infty}(j+1)\left[\frac{1}{k+2}\right]^{j+2}
$$

From Figure 1 it is clear that $I\left(F_{L_{n}}, F\right)$ for exponential distribution with mean $\frac{1}{2}$ is increasing function of $n$ and $\lim _{n \rightarrow \infty} I\left(F_{L_{n}}, F\right)=\frac{1.644}{\lambda}$.

Proposition 2.1. Suppose that $X$ is a non-negative random variable with $c d f F$, then we have

$$
I\left(F_{L_{n}}, F\right)=\int_{0}^{\infty} \sum_{j=0}^{n-1}(j+1)\left[F_{L_{j+2}}(x)-F_{L_{j+1}}(x)\right] d x .
$$

Proof.The proof follows from (1.4) and (2.1). 
Tahmasebi, Nezakati and Daneshi
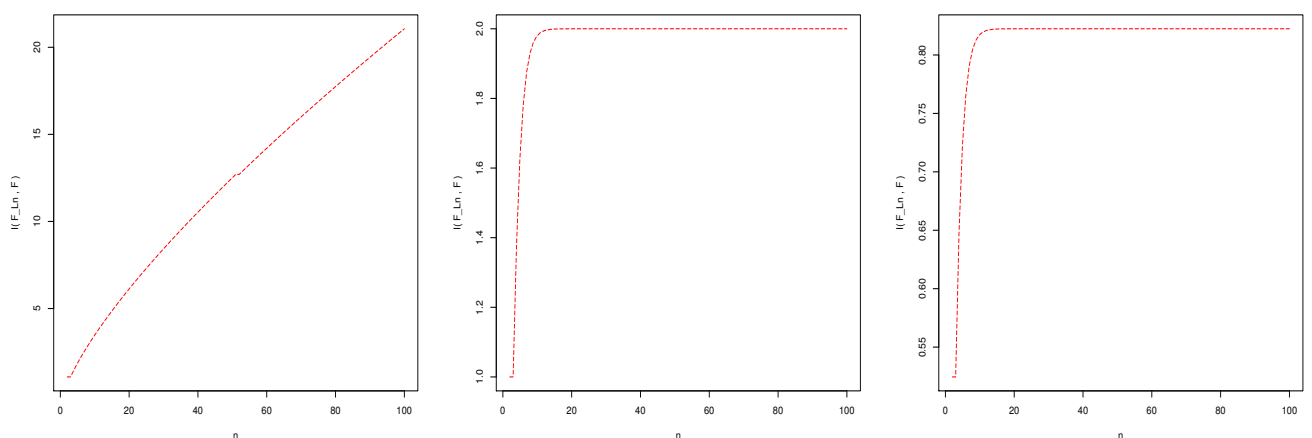

Fig. 1. Plot of $I\left(F_{L_{n}}, F\right)$ in inverse Weibull distribution for $\alpha=\beta=2$ (left), uniform distribution on $(0,1)$ (middle) and exponential distribution with $\lambda=2$ (right).

Proposition 2.2. Let $X$ be a non-negative random variable with $c d f F$, then we have

$$
I\left(F_{L_{n}}, F\right)=\sum_{j=0}^{n-1} \frac{1}{j !} \int_{0}^{\infty} \tilde{\lambda}(z)\left[\int_{0}^{z}[-\log F(x)]^{j} F(x) d x\right] d z
$$

Proof. By (2.1) and the relation $-\log F(x)=\int_{x}^{\infty} \tilde{\lambda}(z) d z$, we have

$$
\begin{aligned}
I\left(F_{L_{n}}, F\right) & =\sum_{j=0}^{n-1} \int_{0}^{\infty} \frac{[-\log F(x)]^{j+1}}{j !} F(x) d x \\
& =\sum_{j=0}^{n-1} \int_{0}^{\infty}\left[\int_{x}^{\infty} \tilde{\lambda}(z) d z\right] \frac{[-\log F(x)]^{j}}{j !} F(x) d x \\
& =\sum_{j=0}^{n-1} \frac{1}{j !} \int_{0}^{\infty} \tilde{\lambda}(z)\left[\int_{0}^{z}[-\log F(x)]^{j} F(x) d x\right] d z .
\end{aligned}
$$

So, the proof is completed.

Proposition 2.3. Let $X$ be a non-negative random variable with $c d f F$, then an analytical expression for $I\left(F_{L_{n}}, F\right)$ is given by

$$
I\left(F_{L_{n}}, F\right)=\sum_{j=0}^{n-1} \int_{0}^{\infty} \frac{[-\log F(x)]^{j+1}}{j !} F(x) d x=\sum_{j=0}^{n-1}(j+1) \mathscr{C}_{j}{ }_{j+1}(X),
$$

where

$$
\mathscr{C} \mathscr{E}_{j+1}(X)=\int_{0}^{\infty} \frac{[-\log F(x)]^{j+1}}{(j+1) !} F(x) d x,
$$

is a generalized cumulative entropy (see Kayal (2016)). 
Proposition 2.4. Let $a, b>0$ for $n=1,2, \ldots$. It holds that

$$
I\left(F_{a L_{n}+b}, F_{a X+b}\right)=a I\left(F_{L_{n}}, F\right) .
$$

Proof. From (2.3), we have

$$
I\left(F_{a L_{n}+b}, F_{a X+b}\right)=\sum_{j=0}^{n-1}(j+1) \mathscr{C} \mathscr{E}_{j+1}(a X+b)=a \sum_{j=0}^{n-1}(j+1) \mathscr{C}_{\mathscr{E}_{j+1}}(X)=a I\left(F_{L_{n}}, F\right)
$$

The proof is completed.

Proposition 2.5. Let $X$ be a absolutely continue non-negative random variable with $I\left(F_{L_{n}}, F\right)<\infty$, for all $n \geq 1$. Then we have

$$
I\left(F_{L_{n}}, F\right)=\sum_{j=0}^{n-1} \frac{1}{j !} E\left(\tilde{h}_{j+1}(T)\right)
$$

where $\tilde{h}_{j+1}(t)=\int_{t}^{\infty}[-\log F(x)]^{j+1} d x$.

Proof. By using (2.1) and Fubini's theorem we obtain

$$
\begin{aligned}
I\left(F_{L_{n}}, F\right) & =\sum_{j=0}^{n-1} \int_{0}^{\infty} \frac{[-\log F(x)]^{j+1}}{j !}\left[\int_{0}^{x} f(t) d t\right] d x \\
& =\sum_{j=0}^{n-1} \int_{0}^{\infty} \frac{f(t)}{j !}\left[\int_{t}^{\infty}[-\log F(x)]^{j+1} d x\right] d t \\
& =\sum_{j=0}^{n-1} \frac{1}{j !} E\left[\tilde{h}_{j+1}(T)\right] .
\end{aligned}
$$

Remark 2.1. Let $X$ be a symmetric random variable with respect to the finite mean $\mu=E(X)$, i.e. $F(x+\mu)=1-F(\mu-x)$ for all $x \in \mathbb{R}$. Then

$$
I\left(F_{L_{n}}, F\right)=I\left(\bar{F}_{R_{n}}, \bar{F}\right)
$$

where $I\left(\bar{F}_{R_{n}}, \bar{F}\right)$ is the cumulative residual measure of inaccuracy between $\bar{F}_{R_{n}}$ (survival function of nth upper record value $R_{n}$ ) and $\bar{F}$.

Kayal (2016) defined the MIT of lower record values as

$$
\mu_{n}(t)=E\left[t-L_{n} \mid L_{n} \leq t\right]=\frac{\int_{0}^{t} F_{L_{n}}(x) d x}{F_{L_{n}}(t)}=\frac{\sum_{j=0}^{n-1} \int_{0}^{t} \frac{F(x)[-\log F(x)]^{j}}{j !} d x}{\sum_{j=0}^{n-1} \frac{F(t)[-\log F(t)]^{j}}{j !}} .
$$

Note that $\mu_{1}(t)=\frac{\int_{0}^{t} F(x) d x}{F(t)}$ is the MIT of the parent distribution. Now we consider a connection between $\mu_{n}(t)$ and $I\left(F_{L_{n}}, F\right)$. 
Tahmasebi, Nezakati and Daneshi

Proposition 2.6. Let $X$ be a non-negative random variable with $c d f F$, then we have

$$
I\left(F_{L_{n}}, F\right)=\sum_{j=0}^{n-1} E\left[\mu_{n}\left(X_{j+1}\right)\right]
$$

Proof. From (2.2) we have

$$
\begin{aligned}
I\left(F_{L_{n}}, F\right) & =\sum_{j=0}^{n-1} \frac{1}{j !} \int_{0}^{\infty} \tilde{\lambda}(z)\left[\int_{0}^{z}[-\log F(x)]^{j} F(x) d x\right] d z \\
& =\int_{0}^{\infty} \tilde{\lambda}(z) \sum_{j=0}^{n-1} \frac{1}{j !}\left[\int_{0}^{z}[-\log F(x)]^{j} F(x) d x\right] d z \\
& =\int_{0}^{\infty} \tilde{\lambda}(z) \mu_{n}(z)\left[\sum_{j=0}^{n-1} \frac{1}{j !}[-\log F(z)]^{j} F(z)\right] d z \\
& =\int_{0}^{\infty} \mu_{n}(z)\left[\sum_{j=0}^{n-1} \frac{1}{j !} f(z)[-\log F(z)]^{j}\right] d z \\
& =\sum_{j=0}^{n-1} \int_{0}^{\infty} \mu_{n}(z) f_{L_{j+1}}(z)=\sum_{j=0}^{n-1} E\left[\mu_{n}\left(X_{j+1}\right)\right] .
\end{aligned}
$$

Hence, the desired result follows.

Proposition 2.7. Suppose that $X$ is a non-negative random variable with $c d f F$, then we have

$$
I\left(F_{L_{n}}, F\right)=\sum_{j=0}^{n-1} \frac{1}{j !}\left[\sum_{i=0}^{j} \frac{1}{i !} E\left[(-\log F(X))^{i} \mu_{j+1}(X)\right]-\sum_{i=0}^{j-1} \frac{1}{i !} E\left[(-\log F(z))^{i} \mu_{j}(X)\right]\right] .
$$

Proof. By using (1.4) and (2.2) we obtain

$$
\begin{aligned}
I\left(F_{L_{n}}, F\right) & =\sum_{j=0}^{n-1} \frac{1}{j !} \int_{0}^{\infty} \tilde{\lambda}(z)\left[\int_{0}^{z}\left[F_{L_{j+1}}(x)-F_{L_{j}}(x)\right] d x\right] d z \\
& =\sum_{j=0}^{n-1} \frac{1}{j !} \int_{0}^{\infty} \tilde{\lambda}(z)\left[\mu_{j+1}(z) F_{L_{j+1}}(z)-\mu_{j}(z) F_{L_{j}}(z)\right] d z \\
& =\sum_{j=0}^{n-1} \frac{1}{j !}\left(\sum_{i=0}^{j} \frac{1}{i !} \int_{0}^{\infty} f(z)[-\log F(z)]^{i} \mu_{j+1}(z) d z\right. \\
& \left.-\sum_{i=0}^{j-1} \frac{1}{i !} \int_{0}^{\infty} f(z)[-\log F(z)]^{i} \mu_{j}(z) d z\right) \\
& =\sum_{j=0}^{n-1} \frac{1}{j !}\left[\sum_{i=0}^{j} \frac{1}{i !} E\left[(-\log F(X))^{i} \mu_{j+1}(X)\right]-\sum_{i=0}^{j-1} \frac{1}{i !} E\left[(-\log F(z))^{i} \mu_{j}(X)\right]\right] .
\end{aligned}
$$

This completes the proof. 
Remark 2.2. Let $X$ be a non-negative random variable with cdf $F$ and $X_{i+1}$ be the $(i+1)$ th lower record with pdf $f_{L_{i+1}}(x)$. Then for $n \geq 1$, we have

$$
I\left(F_{L_{n}}, F\right)=\sum_{j=0}^{n-1} \sum_{i=0}^{j} \frac{1}{j !}\left[E\left[\mu_{j+1}\left(X_{i+1}\right)\right]-E\left[\mu_{j}\left(X_{i+1}\right)\right]\right]
$$

Proof. The proof follows from Proposition 2.7.

Remark 2.3. In analogy with (2.1), a measure of cumulative past inaccuracy associated with $F$ and $F_{L_{n}}$ is given by

$$
I\left(F, F_{L_{n}}\right)=\mathscr{C} \mathscr{E}(X)-E\left[U \log \left(\sum_{j=0}^{n-1} \frac{(-\log U)^{j}}{j !}\right) f\left(F^{-1}(U)\right)\right]
$$

where $\mathscr{C} \mathscr{E}(X)=-\int_{0}^{\infty} F(x) \log F(x) d x$ is the cumulative entropy(see Di Crescenzo and Longobardi (2009)).

In the sequel we obtain upper bound of $I\left(F, F_{L_{n}}\right)$.

Proposition 2.8. Let $X$ be a non-negative random variable that take values in $[0, a]$. Then,

$$
I\left(F, F_{L_{n}}\right) \leq[a-E(X)]\left|\log \left(1-\frac{E\left(L_{n}\right)}{a}\right)\right|
$$

Proof. The proof follows from Proposition 1.9 of Ghosh and Kundu(2017) with the help of log-sum inequality.

In the next propositions we recall some lower bounds for $I\left(F_{L_{n}}, F\right)$.

Proposition 2.9. If $X$ denotes an absolutely continue non-negative random variable with mean $\mu=E X<\infty$. Then for $n \geq 1$, we have

$$
I\left(F_{L_{n}}, F\right) \geq \sum_{j=0}^{n-1} \frac{\tilde{h}_{j+1}(\mu)}{j !}
$$

where the function $\tilde{h}_{j+1}($.$) is defined in Proposition 2.5.$

Proof. From (2.3) we have

$$
\begin{aligned}
I\left(F_{L_{n}}, F\right) & =\sum_{j=0}^{n-1}(j+1) \mathscr{C} \mathscr{E}_{j+1}(X) \\
& =\sum_{j=0}^{n-1} \frac{E\left(\tilde{h}_{j+1}(X)\right)}{j !} .
\end{aligned}
$$


Tahmasebi, Nezakati and Daneshi

Since $\tilde{h}_{j+1}(X)$ is a convex function, applying Jensen's inequality we obtain

$$
I\left(F_{L_{n}}, F\right) \geq \sum_{j=0}^{n-1} \frac{\tilde{h}_{j+1}(\mu)}{j !} .
$$

Proposition 2.10. Let $X$ be a non-negative random variable with $c d f F$, then we have

$$
I\left(F_{L_{n}}, F\right)=\sum_{j=0}^{n-1}(j+1) \mathscr{C} \mathscr{E}_{j+1}(X) \geq \sum_{j=0}^{n-1} \frac{[\mathscr{C} \mathscr{E}(X)]^{j+1}}{j !},
$$

where $\mathscr{C} \mathscr{E}(X)$ is given in Remark 2.3(For more details see Di Crescenzo and Longobardi (2009)).

Proof. Since $(F(x))^{n} \leq F(x)$, for all $n=1,2, \ldots$, we have

$$
\begin{aligned}
I\left(F_{L_{n}}, F\right) & =\sum_{j=0}^{n-1} \int_{0}^{\infty} \frac{(-\log F(x))^{j+1}}{j !} F(x) d x \\
& \geq \sum_{j=0}^{n-1} \int_{0}^{\infty} \frac{(-\log F(x))^{j+1}}{j !}(F(x))^{j+1} \\
& =\sum_{j=0}^{n-1} \int_{0}^{\infty} \frac{[(-\log F(x)) F(x)]^{j+1}}{j !} d x \\
& \geq \sum_{j=0}^{n-1} \frac{1}{j !}\left[\int_{0}^{\infty}(-\log F(x)) F(x) d x\right]^{j+1},
\end{aligned}
$$

which immediately follows (2.9).

Remark 2.4. Let $X$ be a non-negative random variable with $\operatorname{cdf} F$, then for $n=1,2, \ldots$ we have

$$
I\left(F_{L_{n}}, F\right) \geq \sum_{j=0}^{n-1} \frac{1}{j !}\left[\int_{0}^{\infty} F(x) \bar{F}(x) d x\right]^{j+1} .
$$

Proof. By using Proposition 4.3 of Dicresenzo and Longobardi (2009) a lower bound for $\mathscr{C} \mathscr{E}(X)$ is

$$
\mathscr{C} \mathscr{E}(X) \geq \int_{0}^{\infty} F(x) \bar{F}(x) d x .
$$

Now, Proposition 2.10 completes the proof.

Proposition 2.11. For a non-negative random variable and $n=1,2, \ldots$, we have

$$
I\left(F_{L_{n}}, F\right) \geq \sum_{j=0}^{n-1} \frac{1}{j !}[\mu . \operatorname{gini}(X)]^{j+1},
$$

where $\mu=E(X)$ and gini[.] is the Gini index, a celebrated measure of income inequality denoted by(see Wang 1998)

$$
\operatorname{gini}[X]=1-\frac{\int_{0}^{\infty}[\bar{F}(x)]^{2} d x}{E(X)} .
$$

Proof. From Proposition 5.1 of Wang (1998), we have

$$
\int_{0}^{\infty} F(x) \bar{F}(x) d x=\frac{1}{2} E(|X-Y|)
$$




$$
=E(X) \cdot \operatorname{gini}[X],
$$

where $X$ and $Y$ are independent and have the same distributions. Hence, Eq (2.10) completes the proof.

Corollary 2.1. Let $X$ be a non-negative random variable with survival function $\bar{F}(x)$, then we have

$$
I\left(F_{L_{n}}, F\right) \geq \sum_{j=0}^{n-1} \frac{1}{j !} c^{j+1} e^{(j+1) H(X)},
$$

where $c=\exp \left(\int_{0}^{1} \log (x|\log x|) d x\right)=0.2065$ and $H(X)=-\int_{0}^{\infty} f(x) \log f(x) d x$ is the Shannon entropy of $X$.

Proof. The proof follows by recalling (2.9) and Proposition 4.2 of Di Crescenzo and Longob$\operatorname{ardi}(2009)$.

Now we can prove an important property of inaccuracy measure using some properties of stochastic ordering. For that we present the following definitions:

1. The random variable $X$ is said to be smaller than $Y$ according to stochastically ordering (denoted by $\left.X \leq^{s t} Y\right)$ if $P(X \geq x) \leq P(Y \geq x)$ for all $x$. It is known that $X \leq^{s t} Y \Leftrightarrow E(\phi(X)) \leq$ $E(\phi(Y))$ for all increasing functions $\phi$ such that the expectations exist.

2. The random variable $X$ is said to be smaller than $Y$ in likelihood ratio ordering(denoted by $\left.X \leq^{l r} Y\right)$ if $\frac{g(x)}{f(x)}$ is increasing in $x$.

3. A random variable $X$ is said to be smaller than a random variable $Y$ in the decreasing convex order, denoted by $X \leq{ }^{d c x} Y$, if $E(\phi(X)) \leq E(\phi(Y))$ for all decreasing convex functions $\phi$ such that the expectations exist.

4. A non-negative random variable $X$ is said to have decreasing reversed hazard rate DRHR if $\tilde{\lambda}_{F}(x)=\frac{f(x)}{F(x)}$ is decreasing in $x$.

Theorem 2.1. Suppose that the non-negative random variable $X$ is DRHR, then

$$
I\left(F_{L_{n+1}}, F\right)-I\left(F_{L_{n}}, F\right) \leq \sum_{i=1}^{n+1} E_{L_{i}}\left[\frac{1}{\tilde{\lambda}(X)}\right] .
$$

Proof. Let $f_{L_{n}}(x)$ be the pdf of $n$-th lower record value $X_{L_{n}}$. Then, the ratio $\frac{f_{L_{n}}(x)}{f_{L_{n+1}}(x)}=\frac{-n}{\log F(x)}$ is increasing in $x$. Therefore, $X_{n+1} \leq_{l r} X_{n}$, and this implies that $X_{n+1} \leq_{s t} X_{n}$, i.e. $\bar{F}_{n+1}(x) \leq \bar{F}_{n}(x)$ (For more details see Shaked and Shanthikumar(2007,Chapter 1)). This is equivalent (see Shaked and Shanthikumar (2007,p.4)) to have

$$
E\left(\phi\left(X_{n+1}\right)\right) \leq E\left(\phi\left(X_{n}\right)\right),
$$

for all increasing functions $\phi$ such that these expectations exist. Thus, if $X$ is DRHR and $\tilde{\lambda}(x)$ is its reversed hazard rate, then $\frac{1}{\hat{\lambda}(x)}$ is increasing in $x$. As a consequence, from (2.1) we have

$$
\begin{aligned}
I\left(F_{L_{n+1}}, F\right) & =\sum_{j=0}^{n}(j+1) E_{L_{j+2}}\left[\frac{1}{\tilde{\tilde{\lambda}}(X)}\right] \\
& \leq \sum_{j=0}^{n}(j+1) E_{L_{j+1}}\left[\frac{1}{\tilde{\tilde{\lambda}}(X)}\right]
\end{aligned}
$$


Tahmasebi, Nezakati and Daneshi

$$
\begin{aligned}
& =\sum_{i=-1}^{n-1}(i+2) E_{L_{i+2}}\left[\frac{1}{\tilde{\tilde{\lambda}}(X)}\right] \\
& =\sum_{i=0}^{n-1}(i+2) E_{L_{i+2}}\left[\frac{1}{\tilde{\lambda}(X)}\right]+E_{L_{1}}\left[\frac{1}{\tilde{\lambda}(X)}\right] \\
& =I\left(F_{L_{n}}, F\right)+\sum_{i=1}^{n+1} E_{L_{i}}\left[\frac{1}{\tilde{\tilde{\lambda}}(X)}\right] .
\end{aligned}
$$

Thus the proof is completed.

Theorem 2.2. Let $X$ and $Y$ be two non-negative random variables such that $X \leq_{d c x} Y$, then we have

$$
I\left(F_{L_{n}}, F\right) \leq I\left(G_{L_{n}}, G\right) .
$$

Proof. Since $\tilde{h}_{j+1}(x)$ is a decreasing convex function in $x$. Then the proof immediately follows from (2.7).

Proposition 2.12. Let $X$ be a non-negative random variable with absolutely continuous cumulative distribution function $F(x)$. Then for $n=1,2, \ldots$ we have

$$
I\left(F_{L_{n}}, F\right) \geq \sum_{j=0}^{n-1} \sum_{i=0}^{j+1} \frac{(-1)^{i}(j+1)}{i !(j+1-i) !} \int_{0}^{\infty}[F(x)]^{i+1} d x .
$$

Proof. Since $-\log F(x) \geq 1-F(x)$, the proof follows by recalling (2.1).

Proposition 2.13. Let $X$ be a non-negative random variable with absolutely continuous cumulative distribution function $F(x)$. Then for $n=1,2, \ldots$ we have

$$
I\left(F_{L_{n}}, F\right) \leq \sum_{j=0}^{n-1} \frac{1}{j !} \int_{0}^{\infty}[-\log F(x)]^{j+1} d x .
$$

Assume that $\tilde{X}_{\theta}$ denotes a nonnegative absolutely continuous random variable with the distribution function $H_{\theta}(x)=[F(x)]^{\theta}, x \geq 0$. We now obtain the cumulative measure of inaccuracy between $H_{L_{n}}$ and $H$ as follows:

$$
\begin{aligned}
I\left(H_{L_{n}}, H\right) & =-\int_{0}^{+\infty} H_{L_{n}}(x) \log (H(x)) d x \\
& =\sum_{j=0}^{n-1} \theta^{j+1} \int_{0}^{+\infty} \frac{[-\log F(x)]^{j+1}}{j !}[F(x)]^{\theta} d x .
\end{aligned}
$$

Proposition 2.14. If $\theta \geq(\leq) 1$, then for any $n=1,2, \ldots$ we have

$$
I\left(H_{L_{n}}, H\right)=\sum_{j=0}^{n-1}(j+1) \mathscr{C} \mathscr{E}_{j+1}\left(\tilde{X}_{\theta}\right) \leq(\geq) \sum_{j=0}^{n-1} \theta^{j+1}(j+1) \mathscr{C}_{j}{ }_{j+1}(X) .
$$

Proof. Suppose that $\theta \geq(\leq) 1$, then it is clear $[F(x)]^{\theta} \leq(\geq) F(x)$, and hence we have

$$
I\left(H_{L_{n}}, H\right)=\sum_{j=0}^{n-1}(j+1) \mathscr{C} \mathscr{E}_{j+1}\left(\tilde{X}_{\theta}\right) \leq(\geq) \sum_{j=0}^{n-1} \theta^{j+1}(j+1) \mathscr{C} \mathscr{E}_{j+1}(X) .
$$




\section{Dynamic cumulative measure of inaccuracy}

In the reliability theory dynamical measures are useful to describe the information content carried by random lifetimes as age varies. In this section, we study dynamic version of $I\left(F_{L_{n}}, F\right)$. If a system that begins to work at time 0 is observed only at deterministic inspection times, and is found to be down at time $t$, then we consider a dynamic cumulative measure of inaccuracy as

$$
\begin{aligned}
I\left(F_{L_{n}}, F ; t\right) & =-\int_{0}^{t} \frac{F_{L_{n}}(x)}{F_{L_{n}}(t)} \log \left(\frac{F(x)}{F(t)}\right) d x \\
& =\log F(t) \mu_{n}(t)-\int_{0}^{t} \frac{F_{L_{n}}(x)}{F_{L_{n}}(t)} \log (F(x)) d x \\
& =\log F(t) \mu_{n}(t)+\frac{1}{F_{L_{n}}(t)} \sum_{j=0}^{n-1} \int_{0}^{t} \frac{[-\log F(x)]^{j+1}}{j !} F(x) d x .
\end{aligned}
$$

Note that $\lim _{t \rightarrow \infty} I\left(F_{L_{n}}, F ; t\right)=I\left(F_{L_{n}}, F\right)$. Since $\log F(t) \leq 0$ for $t \geq 0$, we have

$$
\begin{aligned}
I\left(F_{L_{n}}, F ; t\right) & \leq \frac{1}{F_{L_{n}}(t)} \sum_{j=0}^{n-1} \int_{0}^{t} \frac{[-\log F(x)]^{j+1}}{j !} F(x) d x \\
& \leq \frac{1}{F_{L_{n}}(t)} \sum_{j=0}^{n-1} \int_{0}^{+\infty} \frac{[-\log F(x)]^{j+1}}{j !} F(x) d x=\frac{I\left(F_{L_{n}}, F\right)}{F_{L_{n}}(t)} .
\end{aligned}
$$

In the following theorem, we prove that $I\left(F_{L_{n}}, F ; t\right)$ uniquely determine the distribution function.

Theorem 3.1. Let $X$ be a nonnegative continuous random variable with distribution function $F($.$) .$ Let the dynamic cumulative inaccuracy of the nth lower record value denoted by $I\left(F_{L_{n}}, F ; t\right)<\infty$, $t \geq 0$. Then $I\left(F_{L_{n}}, F ; t\right)$ characterizes the distribution function.

Proof. From (3.1) we have

$$
I\left(F_{L_{n}}, F ; t\right)=\log F(t) \mu_{n}(t)+\frac{1}{F_{L_{n}}(t)} \sum_{j=0}^{n-1} \int_{0}^{t} \frac{[-\log F(x)]^{j+1}}{j !} F(x) d x .
$$

Differentiating both side of (3.2) with respect to t we obtain:

$$
\begin{aligned}
\frac{d}{d t}\left[I\left(F_{L_{n}}, F ; t\right)\right] & =\tilde{\lambda}_{F}(t) \mu_{n}(t)-\tilde{\lambda}_{F_{L_{n}}}(t) I\left(F_{L_{n}}, F ; t\right) \\
& =\tilde{\lambda}_{F}(t) \mu_{n}(t)-c(t) \tilde{\lambda}_{F}(t) I\left(F_{L_{n}}, F ; t\right) \\
& =\tilde{\lambda}_{F}(t)\left[\mu_{n}(t)-c(t) I\left(F_{R_{n}}, F ; t\right)\right] .
\end{aligned}
$$

Taking derivative with respect to t again we get

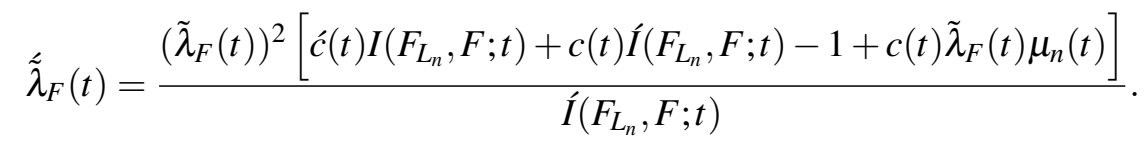


Tahmasebi, Nezakati and Daneshi

Suppose that there are two functions $F$ and $F^{*}$ such that

$$
I\left(F_{L_{n}}, F ; t\right)=I\left(F_{L_{n}}^{*}, F^{*} ; t\right)=z(t) .
$$

Then for all $t$, from (3.3) we get

$$
\tilde{\tilde{\lambda}}_{F}(t)=\varphi\left(t, \tilde{\lambda}_{F}(t)\right), \tilde{\tilde{\lambda}}_{F^{*}}(t)=\varphi\left(t, \tilde{\lambda}_{F^{*}}(t)\right),
$$

where

$$
\varphi(t, y)=\frac{y^{2}[\hat{c}(t) z(t)-c(t) \dot{z}(t)-1+c(t) y w(t)]}{\dot{z}(t)},
$$

and $w(t)=\mu_{n}(t)$. By using Theorem (2.1) and Lemma (2.2) of Gupta and Kirmani(2008), we have $\tilde{\lambda}_{F}(t)=\tilde{\lambda}_{F^{*}}(t)$, for all $t$. Since the reversed hazard rate function characterizes the distribution function uniquely, we complete the proof.

\section{Empirical cumulative measure of inaccuracy}

In this section we study the problem of estimating the cumulative measure of inaccuracy by means of the empirical cumulative inaccuracy in lower record values. Let $X_{1}, X_{2}, \ldots, X_{m}$ be a random sample of size $m$ from an absolutely continuous cumulative distribution function $F(x)$. Then according to (2.3), the empirical cumulative measure of inaccuracy is defined as

$$
\hat{I}\left(F_{L_{n}}, F\right)=\sum_{j=0}^{n-1} \int_{0}^{\infty} \frac{\left[-\log \hat{F}_{m}(x)\right]^{j+1}}{j !} \hat{F}_{m}(x) d x=\sum_{j=0}^{n-1}(j+1) \mathscr{C}_{\mathscr{E}}{ }_{j+1}\left(\hat{F}_{m}\right),
$$

where

$$
\hat{F}_{m}(x)=\frac{1}{m} \sum_{i=1}^{m} I_{\left(X_{i} \leq x\right)}, x \in \mathbb{R} .
$$

is the empirical distribution of the sample and $I$ is the indicator function. If we denote $X_{(1)} \leq X_{(2)} \leq$ $\ldots \leq X_{(m)}$ as the order statistics of the sample, then (4.1) can be written as

$$
\hat{I}\left(F_{L_{n}}, F\right)=\sum_{j=0}^{n-1} \sum_{k=1}^{m-1} \int_{X_{(k)}}^{X_{(k+1)}} \frac{\left[-\log \hat{F}_{m}(x)\right]^{j+1}}{j !} \hat{F}_{m}(x) d x .
$$

Moreover,

$$
\hat{F}_{m}(x)=\left\{\begin{array}{lc}
0, & x<X_{(1)}, \\
\frac{k}{m}, & X_{(k)} \leq x \leq X_{(k+1)}, \quad k=1,2, \ldots, j \\
1, & x>X_{(k+1)} .
\end{array}\right.
$$

Hence, (4.2) can be written as

$$
\hat{I}\left(F_{L_{n}}, F\right)=\sum_{j=0}^{n-1} \sum_{k=1}^{m-1} \frac{1}{j !} U_{k+1} \frac{k}{m}\left(-\ln \frac{k}{m}\right)^{j+1}
$$

where $U_{k+1}=X_{(k+1)}-X_{(k)}, k=1,2, \ldots, m-1$ are the sample spacings. 
Example 4.1. Let $X_{1}, X_{2}, \ldots, X_{m}$ be a random sample drawn from exponential distribution with mean $\frac{1}{\lambda}$. Then the sample spacings $U_{k+1}$ are independent and exponentially distributed with mean $\lambda(m-$ $k$ ) (for more details see Pyke (1965)). Now from (4.3) we obtain

$$
E\left[\hat{I}\left(F_{L_{n}}, F\right)\right]=\frac{1}{\lambda} \sum_{j=0}^{n-1} \sum_{k=1}^{m-1} \frac{1}{j !(m-k)}\left(\frac{k}{m}\right)\left(-\ln \frac{k}{m}\right)^{j+1},
$$

and

$$
\operatorname{Var}\left[\hat{I}\left(F_{L_{n}}, F\right)\right]=\frac{1}{\lambda^{2}} \sum_{j=0}^{n-1} \sum_{k=1}^{m-1} \frac{1}{(j !)^{2}(m-k)^{2}}\left(\frac{k}{m}\right)^{2}\left(-\ln \frac{k}{m}\right)^{2(j+1)}
$$

We have computed the values of $E\left[\hat{I}\left(F_{L_{n}}, F\right)\right]$ and $\operatorname{Var}\left[\hat{I}\left(F_{L_{n}}, F\right)\right]$ for sample sizes $m=10,15,20$, $\lambda=0.5,1,2$ and $n=2,3,4,5$ in Table 1 . We can easily see that $E\left[\hat{I}\left(F_{L_{n}}, F\right)\right]$ is increasing in $m$. Also, we consider that $\operatorname{Var}\left[\hat{I}\left(F_{L_{n}}, F\right)\right]$ is decreasing in $m$.

Example 4.2. Let $X_{1}, X_{2}, \ldots, X_{m}$ be a random sample from a population uniformly distributed in $(0,1)$. Then the sample spacings $U_{k+1}$ are independent of beta distribution with parameters 1 and $m$ (for more details see Pyke (1965)). Now from (4.3) we obtain

$$
E\left[\hat{I}\left(F_{L_{n}}, F\right)\right]=\sum_{j=0}^{n-1} \sum_{k=1}^{m-1} \frac{1}{j !(m+1)}\left(\frac{k}{m}\right)\left(-\ln \frac{k}{m}\right)^{j+1},
$$

and

$$
\operatorname{Var}\left[\hat{I}\left(F_{L_{n}}, F\right)\right]=\sum_{j=0}^{n-1} \sum_{k=1}^{m-1} \frac{1}{(j !)^{2}(m)(m+2)}\left(\frac{k}{m}\right)^{2}\left(-\ln \frac{k}{m}\right)^{2(j+1)} .
$$

We have computed the values of $E\left[\hat{I}\left(F_{L_{n}}, F\right)\right]$ and $\operatorname{Var}\left[\hat{I}\left(F_{L_{n}}, F\right)\right]$ for sample sizes $m=10,15,20$ and

\begin{tabular}{|c|c|c|c|c|c|c|c|c|c|c|c|c|}
\hline & \multicolumn{12}{|c|}{$E\left[\hat{I}\left(F_{L_{n}}, F\right)\right]$} \\
\hline$\alpha$ & 0.5 & 1 & 2 & 0.5 & 1 & 2 & 0.5 & 1 & 2 & 0.5 & 1 & 2 \\
\hline$m$ & \multicolumn{3}{|c|}{$n=2$} & \multicolumn{3}{|c|}{$n=3$} & \multicolumn{3}{|c|}{$n=4$} & \multicolumn{3}{|c|}{$n=5$} \\
\hline 10 & 1.96 & 0.980 & 0.490 & 2.395 & 1.197 & 0.598 & 2.614 & 1.307 & 0.653 & 2.711 & 1.355 & 0.677 \\
\hline 15 & 2.011 & 1.005 & 0.502 & 2.471 & 1.235 & 0.617 & 2.716 & 1.358 & 0.679 & 2.834 & 1.417 & 0.708 \\
\hline 20 & 2.035 & 1.017 & 0.508 & 2.506 & 1.253 & 0.626 & 2.765 & 1.382 & 0.691 & 2.896 & 1.448 & 0.724 \\
\hline & \multicolumn{12}{|c|}{$\operatorname{Var}\left[\hat{I}\left(F_{L_{n}}, F\right)\right]$} \\
\hline$\alpha$ & 0.5 & 1 & 2 & 0.5 & 1 & 2 & 0.5 & 1 & 2 & 0.5 & 1 & 2 \\
\hline$m$ & \multicolumn{3}{|c|}{$n=2$} & \multicolumn{3}{|c|}{$n=3$} & \multicolumn{3}{|c|}{$n=4$} & \multicolumn{3}{|c|}{$n=5$} \\
\hline 10 & 0.252 & 0.063 & 0.015 & 0.291 & 0.072 & 0.018 & 0.306 & 0.076 & 0.0191 & 0.310 & 0.077 & 0.0194 \\
\hline 15 & 0.173 & 0.043 & 0.010 & 0.201 & 0.050 & 0.012 & 0.214 & 0.053 & 0.013 & 0.219 & 0.054 & 0.013 \\
\hline 20 & 0.131 & 0.032 & 0.008 & 0.153 & 0.038 & 0.009 & 0.164 & 0.041 & 0.010 & 0.168 & 0.042 & 0.0105 \\
\hline
\end{tabular}
$n=2,3,4,5$. We can easily see that $E\left[\hat{I}\left(F_{L_{n}}, F\right)\right]$ is increasing in $m$ and $n$. Also, we consider that $\lim _{m \rightarrow \infty} \operatorname{Var}\left[\hat{I}\left(F_{L_{n}}, F\right)\right]=0$.

Table 1. Numerical values of $E\left[\hat{I}\left(F_{L_{n}}, F\right)\right]$ and $\operatorname{Var}\left[\hat{I}\left(F_{L_{n}}, F\right)\right]$ for exponential distribution. 
Tahmasebi, Nezakati and Daneshi

Table 2. Numerical values of $E\left[\hat{I}\left(F_{L_{n}}, F\right)\right]$ and $\operatorname{Var}\left[\hat{I}\left(F_{L_{n}}, F\right)\right]$ for uniform distribution.

\begin{tabular}{|c|cccc|cccc|}
\hline & \multicolumn{4}{|c|}{$E\left[\hat{I}\left(F_{L_{n}}, F\right)\right]$} & \multicolumn{4}{c|}{$\operatorname{var}\left[\hat{I}\left(F_{L_{n}}, F\right)\right]$} \\
\hline$m$ & $\mathrm{n}=2$ & $\mathrm{n}=3$ & $\mathrm{n}=4$ & $\mathrm{n}=5$ & $\mathrm{n}=2$ & $\mathrm{n}=3$ & $\mathrm{n}=4$ & $\mathrm{n}=5$ \\
\hline 10 & 0.437 & 0.581 & 0.660 & 0.697 & 0.014 & 0.019 & 0.021 & 0.022 \\
15 & 0.459 & 0.619 & 0.713 & 0.760 & 0.010 & 0.014 & 0.016 & 0.017 \\
20 & 0.470 & 0.637 & 0.739 & 0.794 & 0.007 & 0.011 & 0.013 & 0.014 \\
\hline
\end{tabular}

\section{Conclusions}

In this paper, we discussed on concept of inaccuracy between $F_{L_{n}}$ and $F$. We proposed a dynamic version of cumulative inaccuracy and studied characterization results of it. It is also proved that $I\left(F_{L_{n}}, F ; t\right)$ can uniquely determine the parent distribution $F$. Moreover, we studied some new basic properties of $I\left(F_{L_{n}}, F\right)$ and $I\left(F_{L_{n}}, F ; t\right)$ such as the stochastic order properties. We also constructed bounds for characterization results of $I\left(F_{L_{n}}, F\right)$. Finally, we estimated the cumulative measure of inaccuracy by means of the empirical cumulative inaccuracy in lower record values. These concepts can be applied in measuring the inaccuracy contained in the associated past lifetime.

\section{References}

[1] Arnold, B.C., Balakrishnan, N., Nagaraja, H.N., 1992. A first course in order statistics. John Wiley and Sons. New York.

[2] Di Crescenzo, A., Longobardi, M., 2009. On cumulative entropies. Journal of Statistical Planning and Inference. 139, 4072-4087.

[3] Ghosh, A., Kundu, C., 2017. Bivariate extension of (dynamic) cumulative residual and past inaccuracy measures. Stat Papers. DOI 10.1007/s00362-017-0917-5.

[4] Gupta, R.C., Kirmani, S.N.U.A., 2008. Characterizations based on conditional mean function. Journal of Statistical Planning and Inference. 138, 964-970.

[5] Kayal, S., 2016. On generalized cumulative entropies. Probability in the Engineering and Informational Sciences.30(4), 640-662.

[6] Kerridge, D.F., 1961. Inaccuracy and inference. J. R. Stat. Soc. Ser. B Stat. Methodol. 23 (1), 184-194.

[7] Pyke, R., 1965. Spacings. Journal of Royal Statistical Society, Series B 27: 395-449.

[8] Shaked, M., Shanthikumar, J.G., 2007. Stochastic orders. Springer, New York.

[9] Thapliyal, R., Taneja, H.C., 2013. A measure of inaccuracy in order statistics. J. Stat. Theory Appl. 12(2), 200-207.

[10] Thapliyal, R., Taneja, H.C., 2015a. Dynamic cumulative residual and past inaccuracy measures. J. Stat. Theory Appl. 14(4), 399-412.

[11] Thapliyal, R., Taneja, H.C., 2015b. On residual inaccuracy of order statistics. Statist. Probab. Lett. 97, 125-131.

[12] Wang, S., 1998. An actuarial index of the right-tail risk. N. Am. Actuar. J. 2, 88-101. 